\title{
Red man syndrome due to accidental overdose of rifampicin
}

Sir,

A 4-year-old boy presented with alleged history of accidental consumption of the entire bottle of rifampicin syrup ( $30 \mathrm{ml}$ bottle, $5 \mathrm{ml} / 100 \mathrm{mg}$ ) i.e. $600 \mathrm{mg}$. The syrup rifampicin was prescribed for his elder sister who had pulmonary tuberculosis. Immediately after consuming the syrup, he developed nausea and abdominal pain. After half an hour, fever, facial flushing, and pruritus appeared, and in the next two hours, rapidly increasing periorbital edema was noticed. There was also history of orange red discoloration of tears and urine. There was no history of convulsions, lethargy, altered sensorium, diarrhea, or bleeding manifestations. On admission, he was alert with a heart rate $110 / \mathrm{min}$. respiratory rate $26 / \mathrm{min}$ and blood pressure 96/60 $\mathrm{mm} \mathrm{Hg}$. Facial flushing, periorbital edema, and urticarial rash were present [Figure 1]. Rest of the general and systemic examination was normal. His complete blood count, serum electrolytes, electrocardiograph, and renal function tests were normal. Liver function tests revealed mild elevation in the transaminase levels. After admission, he continued to pass red urine for one day. His facial flushing, periorbital edema, and urticaria disappeared in one day. He was discharged after 2 days of hospital stay. This can be considered as a probable adverse drug reaction as per causality assessment on Naranjo's scale. ${ }^{[1]}$ On follow-up after one month, he had no complaints, and liver function tests were normal.

Rifampicin is an antibacterial agent, used mainly in the treatment of tuberculosis and is generally well-tolerated. ${ }^{[2]}$ When given in usual doses, fewer than $4 \%$ of patients with tuberculosis have significant adverse reactions. Common side effects include abdominal discomfort, 


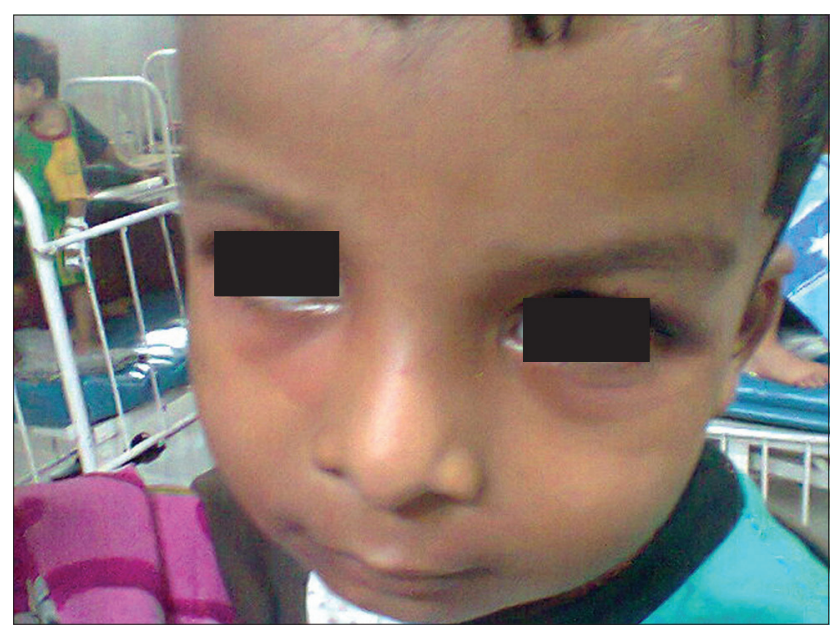

Figure I: Clinical photograph of child showing facial flushing and periorbital oedema

rash, fever, nausea, diarrhea, and vomiting. Rarely, it can also cause acute renal failure, nephrotic syndrome, flulike syndrome, thrombocytopenia, leucopenia, anemia, hemolysis, bleeding manifestations, and elevated serum transaminase levels. Fatigue, drowsiness, headache, dizziness, ataxia, confusion, generalized numbness, pain in extremities, and muscular weakness are some of the adverse reactions on the central nervous system. ${ }^{[2]}$ Adverse reactions are relatively common with intermittent regimens as compared to daily regimens. ${ }^{[2]}$ Cases of accidental overdosage and fatal poisoning have been described. ${ }^{[3]}$ Holdiness et al. studied 29 patients with rifampicin overdosage. ${ }^{[3]}$ Skin discoloration was seen approximately 2.2 hours after administration. Periorbital or facial edema occurred in $72.4 \%$ of the patients, pruritus in $62.1 \%$ and either nausea, vomiting, or diffuse abdominal tenderness in $51.7 \%$. All patients had increased serum bilirubin. A dose of at least $14 \mathrm{gm}$ of rifampicin is necessary before cardiovascular-pulmonary arrest occurs. One of the uncommon adverse effects described with rifampicin include the red man syndrome. Red man syndrome (RMS), an anaphylactoid reaction, is caused by the degranulation of mast cells and basophils, resulting in the release of histamine independent of preformed IgE or complement. ${ }^{[4]}$ The extent of histamine release is related partly to the amount of drug administered. The characteristic signs of RMS include: Orange-red discoloration of the skin, urine, and tears, facial pruritus, and periorbital edema. Although classically described with vancomycin, antibiotics such as ciprofloxacin, amphotericin-B, rifampcin, and teicoplanin can also cause RMS. ${ }^{[4]}$ Like vancomycin, they are capable of causing direct degranulation of mast cells and basophils. RMS is amplified if these antibiotics are combined with vancomycin or with each other. The effects of RMS can be relieved by anti-histamines. In conclusion, pediatricians should be aware of this rare yet easily identifiable syndrome due to rifampicin.

\section{Acknowledgment}

We would like to thank the Dean of our institution for permitting us to publish this manuscript.

\section{Syed Ahmed Zaki, Swapnil Bhongade,} Preeti Shanbag

Department of Pediatrics, Lokmanya Tilak Municipal Medical College and General Hospital, Sion, Mumbai, Maharashtra, India

Correspondence to: Dr. Syed Ahmed Zaki,

Department of Pediatrics, Lokmanya Tilak Municipal Medical College and General Hospital, Sion, Mumbai, Maharashtra, India. E-mail: drzakisyed@gmail.com

\section{References}

1. Naranjo CA, Busto U, Sellers EM, Sandor P, Ruiz I, Roberts EA, et al A method for estimating the probability of adverse drug reactions. Clin Pharmacol Ther 1981;30:239-45.

2. Peter WA. Antimicrobial agents: Drugs used in the chemotherapy of tuberculosis, mycobacterium avium complex disease and leprosy. In: Hardman JG, Limbird LE, editors. Goodman and Gilmans The pharmacological basis of therapeutics. $10^{\text {th }}$ ed. USA: Mc Graw Hill Company; 2001. p. 1273-94.

3. Holdiness MR. A review of the Redman syndrome and rifampicin overdosage. Med Toxicol Adverse Drug Exp 1989;4:444-51.

4. Sivagnanam S, Deleu D. Red man syndrome. Crit Care 2003;7:119-20.

\begin{tabular}{|l|l|}
\hline \multicolumn{2}{|c|}{ Access this article online } \\
\hline Quick Response Code: & Website: \\
\hline & www.jccm.org \\
\cline { 2 - 2 } & DOI: 10.4103/0972-5229.112152 \\
\hline
\end{tabular}

